\title{
Técnicas alternativas de manejo de plantaciones de producción de madera de calidad en régimen intensivo
}

\author{
Alternative management techniques of plantations \\ for the production of quality wood in an intensive regime
}

\author{
López Díaz, M.L.", Moreno, G., Bertomeu, M., Arenas Corraliza, G., Hernández Esteban, A. \\ Grupo de Investigación Forestal, Universidad de Extremadura \\ Avda. Virgen del Puerto $n^{\circ}$ 2, Plasencia - Cáceres 10600, Spain. Tel: 927257000
}

*Autor para correspondencia: lurdesld@unex.es

\section{Resumen}

El empleo de frondosas productoras de madera de calidad se contempla como una vía con grandes perspectivas de mercado futuro. Estas plantaciones se gestionan en régimen intensivo, llevando a cabo una serie de operaciones selvícolas encaminadas a reducir los turnos de corta, pero suponen un coste importante durante largos años en los que no existen ingresos provenientes de la plantación. El control de la vegetación herbácea y la fertilización son dos de las prácticas de manejo más críticas. El manejo silvopastoral y la implantación de praderas de leguminosas podrían permitir reducir los costes económicos de estas plantaciones, así como optimizar las funciones ambientales de las mismas. El objetivo de este ensayo es estudiar la respuesta del nogal en cultivos intensivos (i) con distintas técnicas de control del estrato herbáceo, mecánicas (desbroce y laboreo) y pastoreo (manejo silvopastoral), y (ii) comparando la fertilización tradicional con la implantación de leguminosas. Se han evaluado cómo afectan estos tratamientos a los distintos nutrientes ( $\mathrm{N}, \mathrm{P}, \mathrm{K}$ y Ca) disponibles en suelo y su concentración en hoja. También se han valorado sus implicaciones ambientales mediante la evaluación del control del lixiviado de nitratos. Respecto a las técnicas de control de vegetación, el laboreo trae consigo un empobrecimiento a medio plazo en el sistema. En cambio, el pastoreo puede ser una alternativa más viable gracias al uso más efectivo de los nutrientes que realizan. La presencia de leguminosas en el pasto podría sustituir a medio plazo a los fertilizantes nitrogenados, ya que produce máximos valores de nutrientes en suelo, aunque esto se traduce puntualmente en un mayor lixiviado de nitrato, pero sólo en superficie.

\section{Summary}

The use of broadleaves producing quality wood is seen as a way with great prospects for the future market. These plantations are managed in an intensive regime, carrying out a series of silvicultural operations aimed at reducing the cutting shifts, but they represent an important cost during long years in which there is no income from the plantation. The control of herbaceous vegetation and fertilization are two of the most critical management practices. The silvopastoral management and the implantation of legumes could allow to reduce the economic costs of these plantations, as well as to optimize the environmental functions of the same. The objective of this essay is to study the response of walnut in intensive crops (i) with different control techniques of the herbaceous stratum, mechanics (clearing and tillage) and grazing (silvopastoral management), and (ii) comparing traditional fertilization with implantation of legumes. We evaluated how these treatments affect the different nutrients (N, P, K and Ca) available in soil and their concentration in leaves. Its environmental implications have also been assessed through the evaluation of the control of nitrate leaching. Regarding vegetation control techniques, tillage brings impoverishment in the system at medium-term. On the other hand, grazing can be a more viable alternative thanks to the more effective use of the nutrients. The presence of legumes in the pasture could substitute in the medium term the nitrogen fertilizers, since it produces maximum values of nutrients in soil, although this translates punctually into a greater nitrate leaching, but only at the surface.

Palabras clave: lavado de nitrato, leguminosas, manejo silvopastoral, nogal

Keywords: nitrate wash, legumes, silvopastoral management, walnut 


\section{Introducción}

La gran tasa de importación de madera y derivados en la UE (en torno al 60\%) y en España (alrededor del 40\%), junto con las previsiones de crecimiento del consumo en un futuro próximo y las limitaciones de las extracciones de madera en los bosques tropicales, convierten al sector forestal en estratégico y algunas medidas políticas en el ámbito de la Unión Europea van encaminadas a potenciarlo (McAdam, 2005). Por ello, el empleo de frondosas productoras de madera de calidad se contempla como una vía con grandes perspectivas de mercado futuro.

Estas plantaciones se gestionan en régimen intensivo, llevando a cabo diversas operaciones selvícolas, como son el control de la vegetación competidora y la fertilización, que están encaminadas a incrementar el crecimiento del arbolado, reducir los turnos de corta, e incrementar la calidad de los productos obtenidos y, por tanto, el beneficio final. El principal problema es que estas operaciones suponen un coste importante durante largos años en los que no existen ingresos provenientes de la plantación (Rigueiro-Rodríguez et al., 2009). Pero además, estas prácticas de manejo tienen en muchos casos los costes ambientales propios de la agricultura intensiva (e.g., contaminación difusa por nitratos, fosfatos y herbicidas, empobrecimiento en $\mathrm{C}$ del suelo, pérdida de biodiversidad) (Babcock et al., 2003; The World Bank, 2008).

El método de control más empleado para reducir la competencia de esta vegetación es la aplicación de herbicida combinada con el laboreo, a pesar de su coste tanto económico como medioambiental (McAdam and Sibbald, 2000). La aplicación continuada de herbicidas genera suelos fuertemente minerales, con escaso contenido en materia orgánica y alta dependencia de fertilizantes (Stoate et al., 2001). La implantación de sistemas silvopastorales en esas masas forestales es una práctica de manejo de creciente interés, por mostrar alta eficiencia económica y ambiental (RigueiroRodríguez et al., 2009). En este caso, la vegetación competidora es controlada mediante pastoreo. Éste reduce la utilización de agroquímicos y el riesgo de incendios, a la vez que permite obtener carne de ganado bovino, ovino, equino o caprino.

La utilización de leguminosas como fijadoras de nitrógeno, con incorporación en verde al suelo o simplemente triturada o pastoreada, ha sido ampliamente estudiada y aplicada como mecanismo de fertilización, mostrando generalmente resultados muy satisfactorios. Se trata de una técnica que se ha empleado tradicionalmente en la rotación de los cultivos agrícolas y que actualmente se está poniendo de actualidad como alternativa a los fertilizantes sintéticos (Mccartney and Fraser, 2010). Además, la implantación de praderas ricas en leguminosas permite mejorar el potencial forrajero del sistema.

El objetivo del ensayo es conocer cómo ambas prácticas (implantación de un manejo silvopastoral e implantación de pradera de leguminosas) afectan al ciclo de nutrientes en una plantación de madera de calidad manejada en régimen intensivo. Se plantean las siguientes hipótesis:

a) Ambas prácticas permiten mejorar la fertilidad del suelo, mejorando el estado nutritivo del arbolado. 
b) Ambas prácticas permiten reducir la contaminación difusa por nitrato que generan las plantaciones intensivas.

\section{Material y métodos}

El estudio se realizó en el norte de Extremadura (ETRS89 Zona 20: X: 298.303; Y: 4.442326; 309 m s.n.m.), entre 2011 y 2014 en una plantación de nogal híbrido (Juglans major x nigra 'Mj-209xRa') de 13 años, propiedad de la empresa "Bosques Naturales" (http://bosquesnaturales.com/), con una densidad de 333 pies ha $^{-1}$ y cuyo sustrato inferior se había controlado anteriormente mediante la aplicación de herbicida. La altura y el diámetro normal medios eran $8.33 \mathrm{~m}$ y $17.8 \mathrm{~cm}$, respectivamente. Se desarrolló sobre un suelo franco arenoso, con más de $140 \mathrm{~cm}$ de profundidad y una pendiente menor del $5 \%$. La precipitación y temperatura media de la zona son $952 \mathrm{~mm}$ y $15.6^{\circ} \mathrm{C}$, respectivamente. Los árboles se regaron durante el verano por goteo. Los análisis de suelo iniciales rebelaron un suelo ácido ( $\mathrm{pH} 5$ en agua) y niveles medios de C (2.6\%). Se establecieron dos ensayos (Tab. 1): el primero (ensayo 1) con distintos métodos de control de la vegetación herbácea y el segundo (ensayo 2) basado en la fertilización. Los tratamientos de control de la vegetación bajo el arbolado (ensayo 1) han sido:

a) Labrado en calles y aplicación de herbicidas (glifosato) en las líneas de plantación a principios y mediados de primavera.

b) Desbroce mecánico con motodesbrozadora en las mismas fechas.

c) Introducción de ganado (en pastoreo rotacional durante todo el año excepto verano; 1 ovejas ha ${ }^{-1}$ ). Toda la superficie de ensayo se sometió a un régimen de fertirrigación en verano y la dosis de fertilización característica de la explotación.

El ensayo de fertilización (ensayo 2) consistió en la aplicación de los siguientes tratamientos:

d) No fertilización.

e) Fertilización mineral (aplicación habitual de la explotación, con $40 \mathrm{~kg} \mathrm{~N} \mathrm{Na}^{-1}$, $40 \mathrm{~kg}$ de $\mathrm{P} 2 \mathrm{O} 5 \mathrm{ha}^{-1}$ y $50 \mathrm{~kg} \mathrm{~K} 2 \mathrm{O} \mathrm{ha}{ }^{-1}$ ).

f) Siembra de leguminosa ( $25 \mathrm{~kg} \mathrm{ha}^{-1}$ de Trifolium michelianum y $10 \mathrm{~kg} \mathrm{ha}^{-1} \mathrm{de}$ Ornithopus compressus, en noviembre, tras laboreo, combinada con similares dosis de $\mathrm{P}$ y $\mathrm{K})$.

Todas las áreas de este ensayo se cercaron para impedir el pastoreo hasta finales de primavera, momento en el que se introdujeron ovejas. 
Tabla 1. Descripción de los ensayos y tratamientos.

\begin{tabular}{|c|c|c|}
\hline Ensayos & Tratamientos & Descripción \\
\hline \multirow{3}{*}{$\begin{array}{l}\text { Ensayo 1: Control de la vegetación } \\
\text { (En todas las parcelas se aplicó } \\
\text { fertilizante mineral (NPK) en otoño: } \\
40 \mathrm{~kg} \mathrm{~N} \mathrm{ha}^{-1}, 40 \mathrm{~kg} \mathrm{P}_{2} \mathrm{O}_{5} \mathrm{ha}^{-1} \text { y } 50 \mathrm{~kg} \\
\mathrm{~K}_{2} \mathrm{O} \mathrm{ha}^{-1} \text { ) }\end{array}$} & Desbroce & En otoño y primavera \\
\hline & Laboreo & En otoño y primavera \\
\hline & Pastoreo & - Introduccción de una oveja ha ${ }^{-1}$ \\
\hline \multirow{3}{*}{$\begin{array}{l}\text { Ensayo 2: Fertilización } \\
\text { (Se introdujeron ovejas al final de } \\
\text { primavera después de que secara el } \\
\text { pasto) }\end{array}$} & $\begin{array}{l}\text { Fertilización } \\
\text { inorgánica }\end{array}$ & $\begin{array}{l}\text { - Aplicación de } 40 \mathrm{~kg} \mathrm{~N} \mathrm{ha}^{-1} \\
\quad 40 \mathrm{~kg} \mathrm{P}_{2} \mathrm{O}_{5} \mathrm{ha}^{-1} \text { y } 50 \mathrm{~kg} \mathrm{~K}_{2} \mathrm{O} \mathrm{ha}^{-1}\end{array}$ \\
\hline & $\begin{array}{l}\text { Siembra } \\
\text { de leguminosas }\end{array}$ & $\begin{array}{l}\text { - Aplicación de similares cantidades de PK } \\
\text { - En otoño: laboreo y siembra de } 25 \mathrm{~kg} \mathrm{ha}^{-1} \\
\text { de Trifolium michelanium y } 10 \mathrm{~kg} \mathrm{ha}^{-1} \\
\text { Ornithopus compressus }\end{array}$ \\
\hline & No fertilización & No fertilización ni siembra \\
\hline
\end{tabular}

Se utilizó un diseño de bloques al azar con tres tratamientos en cada ensayo y nueve réplicas, lo que supuso un total de 54 parcelas ( 27 por ensayo). Cada una de estas parcelas $(95 \times 15 \mathrm{~m})$ englobaba dos filas de 20 árboles con sus calles correspondientes a los lados. Las parcelas estaban separadas por una fila de árboles para evitar el efecto borde. Todos los árboles están sometidos a un régimen similar de riego por goteo durante el verano.

Para medir la disponibilidad de nutrientes en suelo, en mayo de 2013 (ensayo 1) y 2014 (ensayo 2), lo que corresponde con el tercer año de cada ensayo, se instalaron verticalmente resinas de intercambio catiónico y aniónico (Plant Root Simulator PRSTM; Ralex), de $45 \mathrm{~cm}^{2}$. Para ello, se hizo un corte vertical con una pala hasta una profundidad de $10-15 \mathrm{~cm}$ de profundidad causando la mínima perturbación posible. A continuación se tapaba. Las resinas instaladas eran de dos tipos: unas para el análisis de $\mathrm{N}$ nítrico $\left(\mathrm{NO}_{3}^{-}\right)$y $\mathrm{P}\left(\mathrm{H}_{2} \mathrm{PO}_{4}^{-}\right)$(resinas de intercambio aniónico), y otras de amonio $\left(\mathrm{NH}_{4}^{+}\right), \mathrm{K}\left(\mathrm{K}^{+}\right)$y Ca $\left(\mathrm{Ca}^{2+}\right)$ (resinas de intercambio catiónico). Se sacaron un mes después y se analizaron en laboratorio los nutrientes indicados tras ser tratadas con $\mathrm{HCl}$ $0.5 \mathrm{~N}(50 \mathrm{~mm})$ durante 1 semana. En junio de 2014, se tomaron muestras de hojas de árbol. Estas muestras se llevaron a laboratorio, donde se realizó el análisis de los principales nutrientes (N, P, K, Ca). Para el estudio de la lixiviación de nitratos, se recogieron muestras del agua del suelo en lisímetros de succión a dos profundidades (30 y $90 \mathrm{~cm}$ ) durante los tres años que duró el ensayo. Se guardaron en nevera nada más recogerlos y se analizaron en las siguientes 24 horas con un espectofotómetro (220 $\mathrm{nm})$. La recogida de muestras, realizada en 12 muestreos desde la noviembre del 2012 hasta abril de 2014, dependió de la climatología.

\section{Resultados}

Los análisis realizados a las resinas de intercambio catiónico y aniónico en los 
ensayos de control de la vegetación (Tab. 2) indicaron que el desbroce consiguió incrementar los niveles de disponibilidad de $\mathrm{P}\left(4.8 \mu \mathrm{g} \mathrm{P} \mathrm{cm}^{-2} \mathrm{mes}^{-1}\right)$, si bien los resultados fueron similares al pastoreo $\left(3.61 \mu \mathrm{g} \mathrm{P} \mathrm{cm}^{-2} \mathrm{mes}^{-1}\right)$. En cambio, con el laboreo se obtuvieron cantidades muy altas de $\mathrm{Ca}\left(64.4 \mu \mathrm{g} \mathrm{Ca} \mathrm{cm}^{-2} \mathrm{mes}^{-1}\right)$ y $\mathrm{N}$ disponible $\left(\mathrm{NO}_{3}{ }^{-}+\mathrm{NH}_{4}{ }^{+}\right)\left(190.3 \mu \mathrm{g} \mathrm{N} \mathrm{cm}^{-2}\right.$ mes $\left.^{-1}\right)$. En cuanto a los tratamientos de fertilización (Tabla 2), la siembra de leguminosas produjo los máximos niveles de $\mathrm{P}, \mathrm{N}$ y K disponibles $\left(5.69 \mu \mathrm{g} \mathrm{P} \mathrm{cm}{ }^{-2} \mathrm{mes}^{-1}, 120.1 \mu \mathrm{g} \mathrm{N} \mathrm{cm}^{-2} \mathrm{mes}^{-1}\right.$ y $80.15 \mu \mathrm{g} \mathrm{K} \mathrm{cm}^{-2}$ mes ${ }^{1}$ ), mientras que en el tratamiento de no fertilización se detectaron los valores máximos de $\mathrm{Ca}\left(60.07 \mu \mathrm{g} \mathrm{Ca} \mathrm{cm}{ }^{-2} \mathrm{mes}^{-1}\right)$.

Respecto al contenido de $\mathrm{N}$ en hoja, no se observaron variaciones significativas. Los mayores valores de este nutriente se observaron con el pastoreo $(22.07 \mathrm{mg}$ $\left.\mathrm{N} \mathrm{g} \mathrm{hoja}{ }^{-1}\right)$, aunque no de forma significativa $(p=0.13)$. En el ensayo de fertilización se comprobó cómo la siembra de leguminosas fue tan efectiva a la hora de aportar $\mathrm{N}$ al árbol (23.34 $\left.\mathrm{mg} \mathrm{N} \mathrm{g}^{-1}\right)$ como la fertilización inorgánica $\left(22.63 \mathrm{mg} \mathrm{N} \mathrm{g}^{-1}\right)$.

Tabla 2. Nutrientes ( $\left.\mathrm{P}, \mathrm{N}, \mathrm{Ca}, \mathrm{K} ; \mu \mathrm{g} \mathrm{cm}^{-2} \mathrm{mes}^{-1}\right)$ disponibles en el suelo y en hoja (N; mg $\mathrm{N} \mathrm{g} \mathrm{hoja} \mathrm{g}^{-1}$ ) tras someter a la masa de nogales a la combinación de distintos tratamientos de control de la vegetación (ensayo 1) y de fertilización (ensayo 2). Se indica el valor medio \pm error estándar. Los valores medios que difieren significativamente se señalan con letras diferentes.

\begin{tabular}{lccccc}
\hline Ensayo 1 & & Desbroce & Laboreo & Pastoreo & sign \\
\hline Suelo & $\mathbf{P}$ & $4.80 \pm 0.89 \mathrm{a}$ & $1.72 \pm 0.51 \mathrm{~b}$ & $3.61 \pm 0.54 \mathrm{ab}$ & $* *$ \\
& $\mathbf{N}$ & $11.33 \pm 1.69 \mathrm{~b}$ & $190.30 \pm 41.85 \mathrm{a}$ & $25.29 \pm 16.11 \mathrm{~b}$ & $* * *$ \\
& $\mathbf{C a}$ & $46.72 \pm 1.88 \mathrm{~b}$ & $64.40 \pm 3.69 \mathrm{a}$ & $52.75 \pm 2.36 \mathrm{~b}$ & $* * *$ \\
& $\mathbf{K}$ & $39.79 \pm 3.83$ & $43.26 \pm 3.31$ & $39.74 \pm 1.81$ & $\mathrm{~ns}$ \\
Hoja & $\mathbf{N}$ & $20.00 \pm 0.77$ & $21.90 \pm 0.65$ & $22.07 \pm 1.13$ & 0.13 \\
\hline Ensayo 2 & & No fertilización & Fertilización & Siembra & \\
\hline Suelo & $\mathbf{P}$ & $1.30 \pm 0.13 \mathrm{~b}$ & $2.57 \pm 0.84 \mathrm{~b}$ & $5.69 \pm 1.31 \mathrm{a}$ & $* *$ \\
& $\mathbf{N}$ & $81.81 \pm 2.70 \mathrm{~b}$ & $88.20 \pm 4.80 \mathrm{~b}$ & $120.10 \pm 6.90 \mathrm{a}$ & $* * *$ \\
& $\mathbf{C a}$ & $60.07 \pm 2.93 \mathrm{a}$ & $52.94 \pm 1.61 \mathrm{~b}$ & $49.69 \pm 1.86 \mathrm{~b}$ & $* *$ \\
& $\mathbf{K}$ & $28.14 \pm 2.00 \mathrm{~b}$ & $44.16 \pm 2.81 \mathrm{~b}$ & $80.15 \pm 14.28 \mathrm{a}$ & $* * *$ \\
Hoja & $\mathbf{N}$ & $21.94 \pm 0.59$ & $22.63 \pm 0.71$ & $23.34 \pm 0.65$ & $\mathrm{~ns}$ \\
\hline
\end{tabular}

En el ensayo de control de la vegetación en nogales (Fig. 1), se observó un incremento de nitrato, aunque tan sólo en superficie ( $30 \mathrm{~cm}$ de profundidad), en las parcelas de laboreo $\left(9.73-39.66 \mathrm{mg} \mathrm{N}-\mathrm{NO}_{3}{ }^{-1} 1^{-1}\right)$, respecto al desbroce $(7.05-14.37 \mathrm{mg}$ $\left.\mathrm{N}-\mathrm{NO}_{3}{ }^{-1} 1^{-1}\right)$ y al pastoreo $\left(6.83-16.59 \mathrm{mg} \mathrm{N}^{-\mathrm{NO}_{3}}{ }^{-1}{ }^{-1}\right)$. Sin embargo, los valores bajaron drásticamente a profundidades mayores $(90 \mathrm{~cm})\left(1-17 \mathrm{mg} \mathrm{N}^{-\mathrm{NO}_{3}}{ }^{-1}{ }^{-1}\right)$, donde no se registraron modificaciones atribuibles a los tratamientos. En la Figura 1 se puede observar la evolución con los distintos tratamientos de fertilización en nogales a 30 y $90 \mathrm{~cm}$ de profundidad. En el año 2013, se observan máximos de nitrato en agua de lixiviado en superficie $(30 \mathrm{~cm})$ con la fertilización inorgánica (entre 


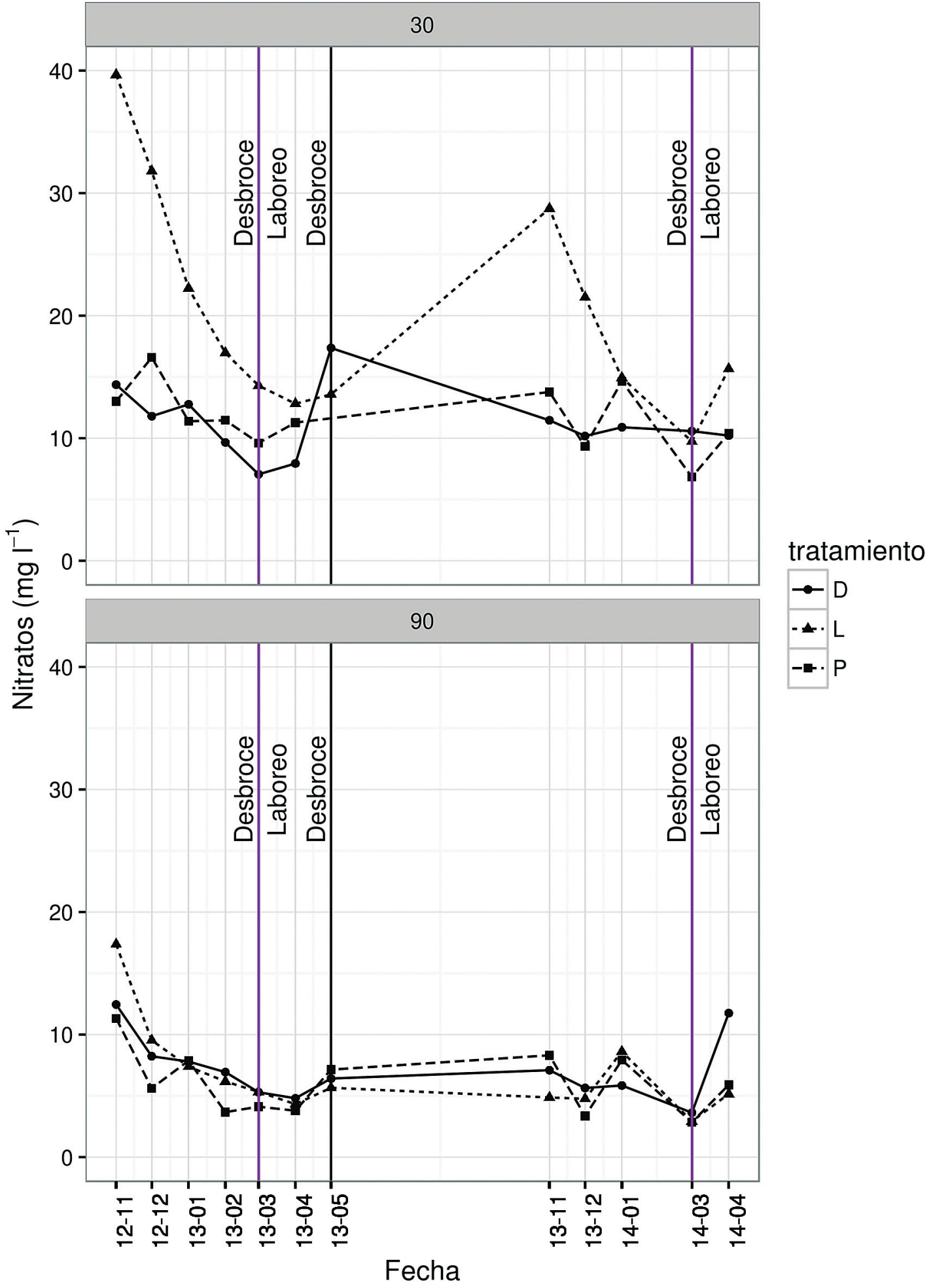




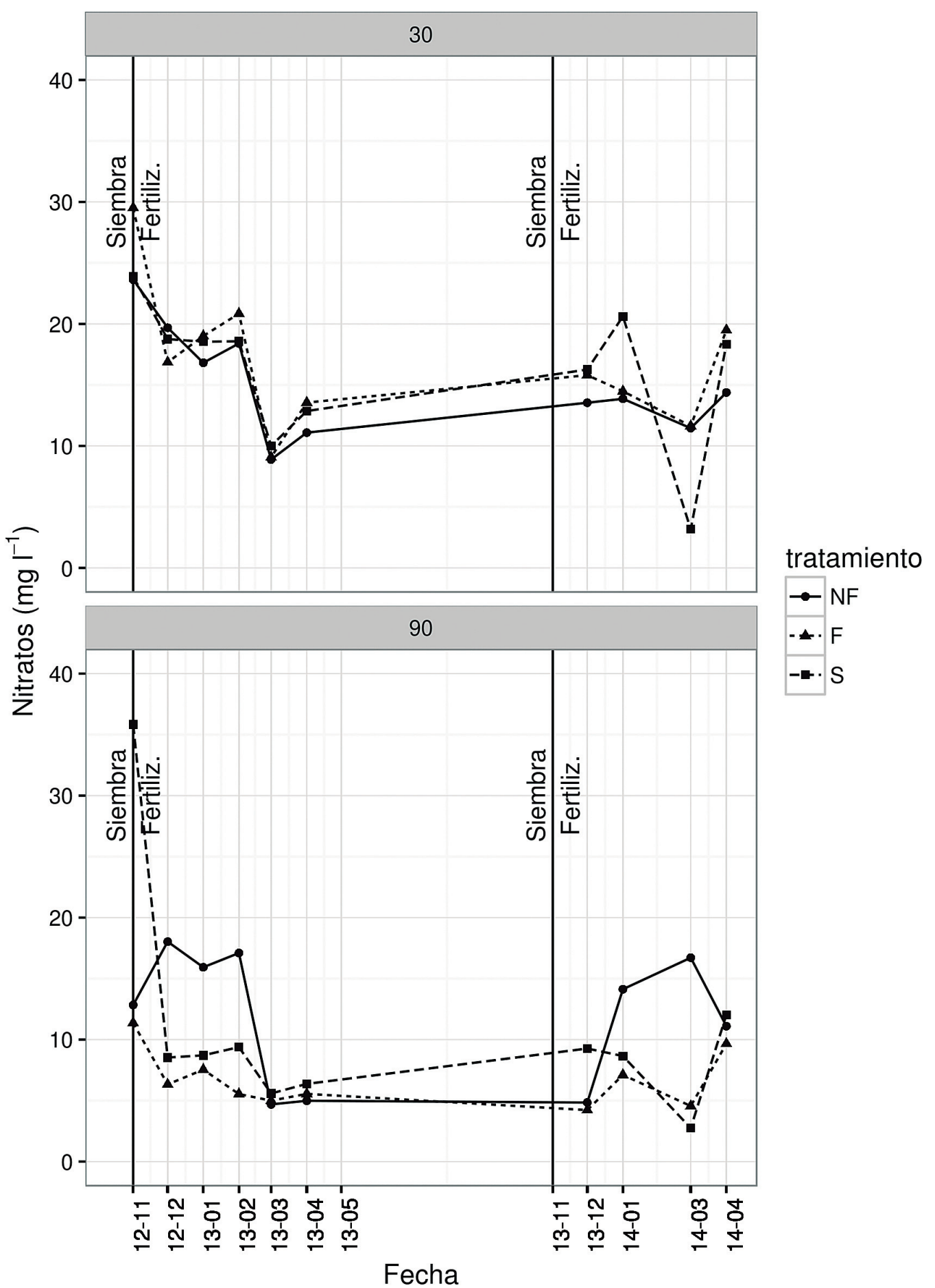

Figura 1. Lavado de nitrato $\left(\mathrm{mg} \mathrm{NO}_{3}^{-1^{-1}}\right)$ bajo los distintos tratamientos de control de la vegetación (izquierda) y fertilización (derecha) en nogal a 30 y $90 \mathrm{~cm}$ de profundidad. L: laboreo; P: pastoreo; D: desbroce; F: fertilización; S: siembra de leguminosas; NF: no fertilización. Las líneas verticales indican la fecha de realización de las distintas labores realizadas en el ensayo de control de la vegetación (ensayo 1) (laboreo y desbroce); y en el ensayo de fertilización (ensayo 2) (fertilización inorgánica NPK; siembra: laboreo, fertilización PK y siembra). 
abril de 2013 y diciembre de 2013 valores de $13.55-15.8 \mathrm{mg} \mathrm{l}^{-1}$ ) y bajo la siembra de leguminosas (20.62 $\mathrm{mg} \mathrm{l}^{-1}$ en enero de 2014). En el muestreo posterior a éste (abril de 2014) los valores bajo las leguminosas bajaron drásticamente. De hecho, el menor valor a $30 \mathrm{~cm}$ se observa bajo la siembra $\left(3.17 \mathrm{mg} \mathrm{l}^{-1}\right.$, frente a los 11.45 $\mathrm{mg}^{-1}$ en el tratamiento de no fertilización y $11.63 \mathrm{mg} \mathrm{l}^{-1}$ en la fertilización). Estas tendencias no se mantienen en profundidad. Los niveles más elevados de nitrato en profundidad se encontraron en las parcelas del tratamiento de no fertilización, sobre todo entre diciembre de 2012 y febrero de $2013\left(17.10 \mathrm{mg} \mathrm{l}^{-1}\right)$ y entre enero y marzo de 2014 (16.71 $\left.\mathrm{mg} \mathrm{l}^{-1}\right)$. En general, el rango de valores detectados en profundidad $(90 \mathrm{~cm})$ es mucho menor que el medido en superficie, excepto los casos señalados del tratamiento no fertilización. También cabe señalar un pico de lavado de nitrato en profundidad $(90 \mathrm{~cm})$ tras el primer laboreo de las parcelas previo a la siembra (en noviembre de 2011: $35.84 \mathrm{mg} \mathrm{l}^{-1}$ ).

\section{Discusión}

El empleo de laboreo como método de control de la vegetación consiguió incrementar la disponibilidad de $\mathrm{N}$ y Ca, debido a la mineralización de la materia orgánica que se produce como consecuencia de la aireación del suelo (Whitehead, 1995). Sin embargo, esta mejora en la nutrición no se reflejó en los nutrientes detectados en las hojas de los árboles, quizás debido a los mayores crecimientos que se observaron en el arbolado (datos no presentados). Además, este aporte de nutrientes que ocurre con el laboreo no se podrá mantener en el tiempo, debido a la reducción en la fuente de nutrientes, como es la materia orgánica, lo que provocará un empobrecimiento del sistema a largo plazo. Esta mayor disponibilidad de $\mathrm{N}$ a corto plazo con el laboreo, unido a la ausencia de vegetación en el estrato inferior, produjo un incremento en el lixiviado de nitratos, lo que podría plantear problemas de contaminación de aguas subterráneas Sin embargo, el hecho de que el aumento de nitratos sólo se detectara en la zona más superficial $(30 \mathrm{~cm})$ y no en la más profunda $(90 \mathrm{~cm})$ reduce el riesgo de contaminación. López-Díaz et al. (2011), en un ensayo realizado en invernadero en el que se comparaba el lavado de nitratos bajo cuatro sistemas distintos de vegetación (suelo desnudo, pasto, arbolado y sistema agroforestal) observaron que bajo los sistemas agroforestales el lavado de nitratos se reducía considerablemente en profundidad, al igual que obtuvieron (Van Der Salm et al., 2006), gracias principalmente a la presencia del arbolado, que es capaz de aprovechar el nitrógeno tanto a nivel superficial, donde también se desarrollan las raíces de las herbáceas, como en capas más profundas (Moreno et al., 2005).

El pastoreo consiguió mejorar ligeramente el contenido de $\mathrm{N}$ en hoja, aunque este contenido no se vio reflejado en el suelo. Se trata, por tanto, de un tratamiento más progresivo pero también efectivo. Es importante señalar que en las zonas en las que se introdujeron animales (pastoreo) no se detectaron incrementos en el lavado nitrógeno, ya que se trata de aportes en su mayor parte orgánicos y, por tanto, disponibles de forma más progresiva (Kramer et al., 2006). 
La siembra de leguminosas mejoró la disponibilidad de $\mathrm{P}, \mathrm{K}$ y $\mathrm{N}$ en el suelo. Según Whitehead (1995), en algunos casos las especies de esta familia pueden fijar entre 0 y $500 \mathrm{~kg} \mathrm{~N} \mathrm{ha}^{-1}$. Estos aportes se producen gradualmente, con lo que se reducen significativamente los problemas de contaminación por nitratos respecto a los fertilizantes inorgánicos (Marinari et al., 2010). El incremento en el nitrato en agua seguramente fue debido también al laboreo que se realizó en invierno previo a la siembra, pero no se mantuvo en el tiempo. En el caso del tratamiento laboreo, este incremento sólo se observó en esos primeros centímetros de suelo. Con la siembra de leguminosas, la concentración de nitrato se mantuvo en profundidad. La diferencia entre ambos puede ser debida a que en noviembre existe una parada vegetativa, con lo que el nitrato no es aprovechado por la vegetación. En cambio, el tratamiento laboreo se aplicó mucho después (en la primavera), en plena actividad vegetativa, aunque es esperable que este trasvase se detecte a medio plazo. También se han observado valores superiores al resto de los tratamientos en donde se había aplicado el fertilizante inorgánico, como consecuencia del aporte de nitrógeno mineral que se produce con este tratamiento, si bien tan sólo se detectó a nivel superficial $(0-30 \mathrm{~cm})$.

Por otro lado, los valores máximos de Ca obtenidos con el tratamiento de no fertilización coinciden con las mínimas producciones tanto en el estrato arbóreo como herbáceo. Se trata de un suelo ácido (pH 5 en agua), con lo que los contenidos de Ca son escasos, y un incremento en la productividad puede reducir esos valores todavía más, por lo que sería conveniente realizar aportes de caliza.

En general, los valores de nitrato en agua lixiviada $\left(2.8-39.7 \mathrm{mg} \mathrm{NO}_{3}^{-1} 1^{-1}\right)$ se encontraban por debajo de la normativa de la Unión Europea, que limita a $50 \mathrm{mg}$ $\mathrm{NO}_{3}{ }^{-1}{ }^{-1}$ la presencia de nitratos en agua potable.

\section{Conclusiones}

En las plantaciones destinadas a la producción de madera de calidad en régimen intensivo tanto el pastoreo, como método de control de la vegetación competidora con el estrato arbóreo, como la siembra de leguminosas, destinadas al aporte de $\mathrm{N}$, son opciones adecuadas tanto a medio como a largo plazo desde el punto de vista productivo y medioambiental.

\section{Agradecimientos}

Este trabajo ha sido financiado por el Plan Nacional I+D+I del Ministerio de Ciencia e Innovación (AGL2011-25456). Se ha realizado en colaboración con la empresa Bosques Naturales SA que ha cedido sus plantaciones de nogal de la provincia de Cáceres además de colaborado en la implantación de los distintos tratamientos ensayados. 


\section{Bibliografía}

Caro Babcock, B., Fraser, R., Lekakis, J., 2003. Risk Management and the Environment: Agriculture in Perspective. Agricultural Economic Series. Kluwer Academic Publisher. https://doi.org/10.1007/978-94-017-2915-4

European Union, 1991. Council Directive of 12th December 1991 concerning the protection of waters against pollution caused by nitrates from agricultural sources (91/676/ EEC). Off. J. Eur. Communities No L 375/1, 1-8.

Kramer, S.B., Reganold, J.P., Glover, J.D., Bohannan, B.J.M., Mooney, H., 2006. Reduced nitrate leaching and enhanced denitrifier activity and efficiency in organically fertilized soils. Proc. Natl. Acad. Sci. 103, 4522-4527. https://doi.org/10.1073/pnas.0600359103

López-Díaz, M.L., Rolo, V., Moreno, G., 2011. Trees' role in nitrogen leaching after organic, mineral fertilization: a greenhouse experiment. J. Environ. Qual. 40, 853-859. https:// doi.org/10.2134/jeq2010.0165

Marinari, S., Lagomarsino, A., Moscatelli, M., Di Tizio, A., Campiglia, E., 2010. Soil carbon and nitrogen mineralization kinetics in organic and conventional three-year cropping systems. Soil Till. Res. 109, 161-168. https://doi.org/10.1016/j.still.2010.06.002

McAdam, J., 2005. Silvopastoral systems in North-west Europe. In: Mosquera-Losada, M.R., McAdam, J., Rigueiro-Rodríguez, A. (eds.). Silvopastoralism and Sustainable Land Management. CABI Publishing, Wallinford (UK), pp. 19-23. https://doi.org/10. 1079/9781845930011.0019

McAdam, J., Sibbald, A., 2000. Grazing livestock management. Forestry Commission Bulletin 122, 44-57.

Mccartney, D., Fraser, J., 2010. The potential role of annual forage legumes in Canada: A review. Can. J. Plant Sci. 90, 403-420. https://doi.org/10.4141/CJPS07182

Moreno, G., Obrador, J.J., Cubera, E., Dupraz, C., 2005. Fine Root Distribution in Dehesas of Central-Western Spain. Plant Soil 277, 153-162. https://doi.org/10.1007/s11104005-6805-0

Rigueiro-Rodríguez, A., Fernández-Nú-ez, E., González-Hernández, P., McAdam, J., Mosquera-Losada, M., 2009. Agroforestry Systems in Europe: Productive, Ecological and Social Perspectives. In: Rigueiro-Rodríguez, A., McAdam, J.M. (eds.), Agroforestry in Europe. Springer, pp. 43-65. https://doi.org/10.1007/978-1-4020-8272-6

Stoate, C., Boatman, N., Borralho, R., Rio Carvalho, C., De Snoo, G., Eden, P., 2001. Ecological impacts of arable intensification in Europe. J. Environ. Manage. 63, 337-365. https://doi.org/10.1006/jema.2001.0473

The World Bank, 2008. Agriculture for Development.

Van Der Salm, C., Denier Van Der Gon, H., Wieggers, R., Bleeker, A., Van Den Toorn, A., 2006. The effect of afforestation on water recharge and nitrogen leaching in The Netherlands. For. Ecol. Manage. 221, 170-182. https://doi.org/10.1016/j.foreco.2005. 09.027

Whitehead, D., 1995. Grassland nitrogen. CAB International 